\section{Two of the usual suspects, platelet-activating factor and its receptor, implicated in acute lung injury}

\author{
Stephen M. Prescott, Thomas M. McIntyre, and Guy Zimmerman
}

Commentary

See related article

in this issue, pages

1071-1076.

Huntsman Cancer Institute and Program in Human Molecular Biology \& Genetics, University of Utah, Salt Lake City, Utah 84112, USA

Address correspondence to: Stephen M. Prescott, Huntsman Cancer Institute, 2000 East Circle of Hope, University of Utah, Salt Lake City, Utah 84112-5550, USA. Phone: (801) 585-3401; Fax: (801) 585-6345; E-mail: steve.prescott@hci.utah.edu.

In this issue of the JCI, Nagase et al. show that the receptor for platelet-activating factor is a crucial component in acute lung injury (ALI) induced by hydrochloric acid in mice (1). In humans, ALI and the adult respiratory distress syndrome (ARDS) are physiologically defined conditions in a spectrum of lung damage that occurs frequently and has a high mortality rate (2). ARDS results from a diverse set of clinical insults. Acid injury following aspiration of gastric contents is a common initiating factor, as are sepsis and trauma $(2,3)$. No specific therapy is available either to prevent ARDS or to hasten its resolution; the standard clinical response - supportive therapy, including supplemental oxygen, assisted ventilation, and treatment of coincident infection - has reduced the mortality rate from $70-80 \%$ a decade ago to the current $30-40 \%$. Nonetheless, mortality and morbidity remain unacceptable, as does the cost of care, much of which is delivered in the intensive care unit.

Inflammation is a cardinal feature of clinical and experimental ALI, as is pulmonary edema resulting from increased permeability. In animal models, depletion of blood polymorphonuclear neutrophils (PMNs) protects against ALI, implicating them as a causal factor. The chemical mediators that initiate the inflammation and alter vascular permeability are not yet identified. Many suspects have been interrogated, and it is highly likely that several villains act in an inflammatory conspiracy in ALI. Prominent among these suspects is platelet-activating factor (PAF). This bioactive phospholipid increases vascular permeability, attracts leukocytes, and primes and triggers their secretory responses (4), properties that raise suspicion but do not suffice to implicate PAF in the genesis of ALI.

Unfortunately, PAF is difficult to analyze, and it is rapidly degraded by tissue and plasma PAF acetylhydrolases (5). Thus, finding elevated levels in the appropriate circumstances has been challenging, although provocative data have been reported (reviewed in ref. 4). Another way to implicate PAF would be to block the receptor through which it signals. Many, but not all, studies that applied this strategy support the conclusion that PAF is important in ALI (4). The absence of specific inhibitors of PAF biosynthesis puts one pharmacological approach to the question out of reach, but the converse approach increasing the rate of degradation of PAF by supplying exogenous PAF acetylhydrolase (5) - remains workable. If PAF is central in an inflammatory syndrome, this enzyme should minimize the damage caused by acid inhalation or other stimuli (6). Currently, recombinant plasma PAF acetylhydrolase is being tested in clinical trials to determine if it can prevent ARDS in high-risk patients.

Nagase et al. (appearing in this issue) used mice that overexpress the PAF receptor (7), and others that lack it (8), to test the hypothesis that PAF is involved in lung injury. In the current work, they injured the lungs with hydrochloric acid and found that control mice died rapidly, after developing inflammation, pulmonary edema, and impaired oxygenation. In animals that overexpressed the PAF receptor, the response was amplified. Conversely, animals that lacked a PAF receptor did not die during the experiment and had much better oxygenation and little edema. These findings demonstrate the importance of the PAF receptor in ALI pathogenesis and constitute a smoking gun that implicates PAF or PAF-like compounds (9) in this process. Nagase et al. made another remarkable observation as well: although the lung injury was prevented in PAF receptor-deficient mice, PMNs accumulated in the
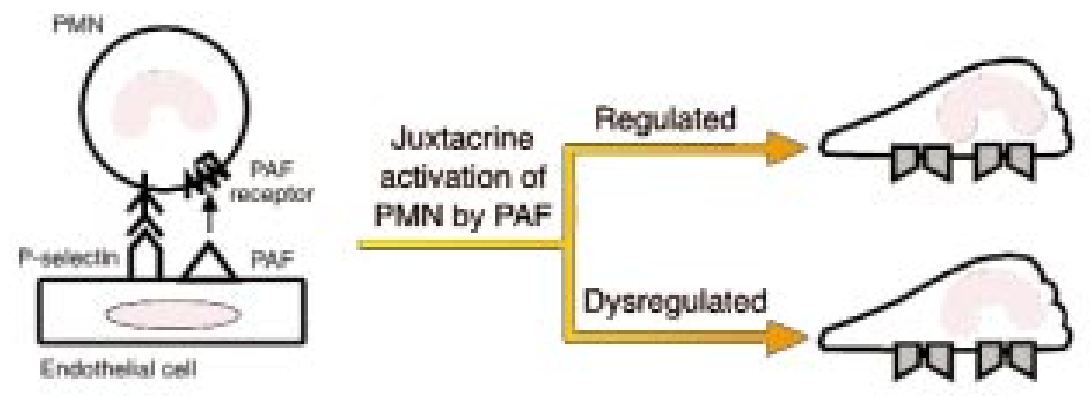

Polarization, $\beta_{2}$ integrin activation, Priming in defensive intlammation

Inflammatory injury in ALI, other syndromes

Figure 1

Juxtacrine signaling by PAF mediates PMN activation. PAF displayed on the plasma membranes of inflamed endothelial cells delivers an activating signal to PMNs that are tethered to the endothelial surface by P-selectin (9). This signal, mediated by the PAF receptor, primes PMNs for enhanced granular secretion and oxygen radical generation, among other inflammatory responses (3). Under normal regulation, this multistep adhesion and signaling pathway contributes to host defense. The same molecular events, when dysregulated or uncontrolled, can induce ALI and other syndromes of inflammatory tissue injury. 
lungs in these animals as in controls. This finding is consistent with other work that suggests that PMN accumulation in the rodent lung occurs by several mechanisms and does not always result in lung injury (3).

These experiments support a model of the molecular events in early inflammation that we proposed previously $(10,11)$, in which PMNs bind to activated endothelial cells in a several-step process. In the first step, PMNs become tethered to P-selectin or other endothelial cell surface selectins. Based on our in vitro studies (Figure 1), as well as subsequent in vivo work (12), PMNs are not activated - at least not fully - at this stage but require a second stimulus if they are to secrete proteolytic enzymes and oxygen radicals or to undergo other inflammatory responses. PAF displayed by endothelial cells under these circumstances can provide this second signal to the PMN, leading to its activation. This juxtacrine model predicts the findings of Nagase et al. that the accumulation of PMNs in the lungs does not require PAF but that subsequent activation is blunted in the absence of the PAF receptor. Mediators other than PAF, such as IL-8 (13), may provide additional activation signals in acid-induced ALI, but the current studies indicate that signaling via the PAF receptor is critical in priming PMNs for enhanced granule secretion, oxidant generation, and cytokine synthesis (4).
Indeed, signaling through this receptor seems to be a limiting factor in ALI pathogenesis, since transgenic animals that overexpress the PAF receptor exhibit heightened inflammatory responses to acid.

The juxtacrine model, which requires both tethers and signals, suggests that cell surface molecules cooperate to activate target cells. This 2 -part mechanism - bringing cells into proximity by an adhesive interaction, and then activating the target cell with a mediator derived from the initiating cell - may provide a general model for juxtacrine signaling. One implication of such a model is that the local concentration of the mediator could be very high, despite having a low systemic concentration. Furthermore, because signaling occurs in a protected environment between 2 cells, the access of drugs such as receptor antagonists may be limited. If this model reflects a common mode by which small-molecule mediators exert their actions, then strategies such as those employed by Nagase et al. will continue to be necessary to test the involvement of a specific small molecule in an intercellular interaction. That is, to implicate the suspected mediator, one must interrogate its receptor.

1. Nagase, et al. 1999. Platelet-activating factor mediates acid-induced lung injury in genetically engineered mice. J. Clin. Invest. 104:1071-1076. 2. Hudson, L.D., and Steinberg, K.P. 1999. Epidemiology of acute lung injury and ARDS. Chest.
116:74S-82S.

3. Matthay, M.A., and Rosen, G.D. 1996. Acid aspiration induced lung injury. New insights and therapeutic options. Am. J. Respir. Crit. Care Med. 154:277-278

4. Prescott, S.M., McIntyre, T.M., and Zimmerman, G.A. 1999. Platelet-activating factor: a phospholipid mediator of inflammation. In Inflammation: basic principles and clinical correlates. J.I. Gallin and R. Snyderman, editors.Lippincott Williams \& Wilkins. Philadelphia, PA. 387-389.

5. Stafforini, D.M., McIntyre, T.M., Zimmerman, G.A., and Prescott, S.M. 1997. Platelet-activating factor acetylhydrolases. J. Biol. Chem. 272:17895-17898.

6. Tjoelker, L.W., et al. 1995. Anti-inflammatory properties of a platelet-activating factor acetylhydrolase. Nature. 374:549-553.

7. Ishii, S., et al. 1997. Bronchial hyperreactivity, increased endotoxin lethality and melanocytic tumorigenesis in transgenic mice overexpressing platelet-activating factor receptor. $E M B O \mathrm{~J}$. 16:133-142.

8. Ishii, S., et al. 1998. Impaired anaphylactic responses with intact sensitivity to endotoxin in mice lacking a platelet-activating factor receptor. J. Exp. Med. 187:1779-1788.

9. McIntyre, T.M., Zimmerman, G.A., and Prescott, S.M. 1999. Biologically active oxidized phospholipids. J. Biol. Chem. 274:25189-25192.

10. Lorant, D.E., et al. 1991. Coexpression of GMP140 and PAF by endothelium stimulated by histamine or thrombin: a juxtacrine system for adhesion and activation of neutrophils. J. Cell Biol. 115:223-234.

11.Zimmerman, G.A., Prescott, S.M., and McIntyre, T.M. 1992. Endothelial cell interactions with granulocytes: tethering and signaling molecules. Immunol. Today. 13:93-100.

12. Coughlan, A.F., Hau, H., Dunlop, L.C., Berndt M.C., and Hancock, W.W. 1994. P-selectin and platelet-activating factor mediate initial endotoxin-induced neutropenia. J. Exp. Med. 179:329-334.

13. Folkesson, H.G., Matthay, M.A., Hebert, C.A., and Broaddus, V.C. 1995. Acid aspiration-induced lung injury in rabbits is mediated by interleukin8-dependent mechanisms. J. Clin. Invest. 96:107-116. 